\title{
Descoloração de efluentes aquosos sintéticos e têxtil contendo corantes índigo e azo via processos Fenton e foto-assistidos (UV e UV/ $\mathrm{H}_{2} \mathrm{O}_{2}$ )
}

\section{Decolorization of synthetic and laundry wastewater containing indigo and azo dyes by the Fenton, photolytic and UV/ $\mathrm{H}_{2} \mathrm{O}_{2}$ processes}

\author{
Bruno César Barroso Salgado \\ Tecnólogo em Processos Químicos pelo Centro Federal de Educação Tecnológica do Ceará (CEFETCE). \\ Pós-graduação em Tecnologia e Gestão Ambiental (PGTGA) pelo CEFETCE \\ Maria Ionete Chaves Nogueira \\ Mestre em Química Inorgânica pela Universidade Federal do Ceará (UFC). Pós-graduação em Saneamento Ambiental \\ do Departamento de Engenharia Hidráulica e Ambiental (DEHA/UFC) \\ Kelly Araújo Rodrigues \\ Doutora em Hidráulica e Saneamento pela Escola de Engenharia de São Carlos da Universidade de São Paulo (EESC/USP). \\ Professora da Área de Química e Meio Ambiente pelo CEFETCE \\ Glória Maria Marinho Silva Sampaio \\ Doutora em Hidráulica e Saneamento pela EESC/USP. Professora da Área de Química e Meio Ambiente pelo CEFETCE
}

Hugo Leonardo de Brito Buarque

Doutor em Física pela UFC. Professor da Área de Química e Meio Ambiente pelo CEFETCE

Rinaldo dos Santos Araújo

Doutor em Química Inorgânica pela UFC. Professor da Área de Química e Meio Ambiente pelo CEFETCE

\begin{abstract}
Resumo
No presente trabalho, processos de oxidação avançada, $\mathrm{Fe}^{2+} / \mathrm{H}_{2} \mathrm{O}_{2}$ e $U \mathrm{~V} / \mathrm{H}_{2} \mathrm{O}_{2}$, e de fotólise (UV) foram empregados na descoloração de dois efluentes sintéticos, contendo corantes tipo índigo e azo, e de um efluente de lavanderia industrial. Experimentalmente, soluções em concentração de 20 mg/L dos corantes índigo carmim e vermelho congo, respectivamente $43 \mu \mathrm{mol} / \mathrm{L}$ e $29 \mu \mathrm{mol} / \mathrm{L}$, e o efluente têxtil $(\mathrm{pH}=3)$ foram submetidos a diferentes condições oxidantes sob temperatura ambiente $\left(27^{\circ} \mathrm{C}\right)$. As remoções de cor e de DQO foram avaliadas em cada sistema oxidativo estudado. Em geral, os resultados obtidos mostraram que os processos utilizados são muito promissores na descoloração dos efluentes. A descoloração completa das soluções foi alcançada nos processos Fenton e com $U V / \mathrm{H}_{2} \mathrm{O}_{2}$. Estudos cinéticos revelam que a taxa de descoloração em meio aquoso segue uma cinética de pseudo-primeira ordem em relação à concentração do corante.
\end{abstract}

Palavras-chave: corantes sintéticos; efluente têxtil; processos de oxidação avançada; descoloração; cinética.

\section{Abstract}

In the present work, advanced oxidation processes, $\mathrm{Fe}^{2+} / \mathrm{H}_{2} \mathrm{O}_{2}$ and $\mathrm{UV} / \mathrm{H}_{2} \mathrm{O}_{2}$, and direct photolysis (UV light) have been applied in the decolorization of two synthetic wastewater containing indigo and azo dyes and laundry effluent. Individual aqueous solutions containing $20 \mathrm{mg} / \mathrm{L}$ indigo carmine and congo red dyes (43 $\mu \mathrm{mol} / \mathrm{L}$ and $29 \mu \mathrm{mol} / \mathrm{L}$, respectively) and textile laundry wastewater at $\mathrm{pH} 3$ were subjected to different experimental conditions in the oxidation reactions at room temperature $\left(27^{\circ} \mathrm{C}\right)$. Color and COD removals were evaluated for each oxidation systems. The results showed that the utilized processes are able to successfully decolorize the wastewaters. Complete bleaching was achieved by Fenton and $\mathrm{UV} / \mathrm{H}_{2} \mathrm{O}_{2}$. Also, kinetics investigations revealed that the decolorization follows pseudo-first order kinetic with respect to the dye concentration.

Keywords: synthetic dyes; textile wastewater; advanced oxidation processes; decolorization; kinetic.

Endereço para correspondência: Rinaldo dos Santos Araújo - Gerência de Química e Meio Ambiente do CEFETCE - Avenida 13 de Maio, 2.081 - Benfica - 60411-220, Fortaleza (CE), Brasil - Tel.: (85) 3307-3611 - Fax: (85) 3307-3633 - E-mail: rinaldo@cefetce.br

Recebido: 30/01/08 - Aceito: 10/12/08 


\section{Introdução}

A poluição do meio ambiente por efluentes industriais tem aumentado gradativamente nas últimas décadas, tornando-se um grave problema social e ambiental. Os resíduos produzidos, em geral, de composição diversificada, frequentemente contêm poluentes tóxicos e resistentes aos sistemas convencionais de tratamento (coagulação/floculação, adsorção com carvão ativado, precipitação, degradação biológica etc.). A indústria têxtil, em particular, utiliza elevada demanda de água em seus processos, gerando grande quantidade de águas residuárias, as quais, geralmente, contêm altas cargas de sais dissolvidos, surfactantes, sólidos suspensos e matéria orgânica, principalmente na forma de moléculas corantes complexas (NEAMTU et al, 2002; ROTT; MINKE, 1999). As substâncias corantes contribuem significativamente para a poluição de recursos hídricos, por dificultarem a penetração dos raios solares, prejudicando o metabolismo fotossintético de algumas espécies; além disso, apresentam-se como recalcitrantes e potencialmente cancerígenas (PETERNEL. KOPRIVANAC, KUŠIĆ, 2006). Segundo Coughlin, Kinkle e Bishop (2003), cerca de 4\% da produção de corantes orgânicos decorrente dos processos de síntese e aplicação se perde para o meio ambiente, produzindo águas residuárias fortemente coloridas. O desenvolvimento de tecnologias eficientes e baratas para o tratamento de efluentes contendo corantes constitui uma necessidade, e consideráveis esforços estão sendo dedicados a esse campo de pesquisa (ZAZO et al, 2006). Neste contexto, processos oxidativos avançados (POAs) se apresentam como tecnologias capazes de reduzir os problemas ambientais gerados por estes efluentes (FORGACS; NANSHENG; HELIN, 2004; SHU; CHANG, 2005). POAs se destacam por poderem efetivamente ser usados na eliminação (mineralização) de componentes tóxicos e danosos, uma vez que destroem as moléculas orgânicas poluentes, ao invés de simplesmente removê-las para outra fase. O termo "POA" é usado para descrever os processos oxidativos em que radicais hidroxilos $(\cdot \mathrm{OH})$ altamente reativos atuam como oxidantes principais (POULOPOULOS; ARVANITAKIS; PHILIPPOPOULOS, 2006; KUŠIĆ et al, 2007). O peróxido de hidrogênio $\left(\mathrm{H}_{2} \mathrm{O}_{2}\right)$ é a fonte mais comum de radicais $\bullet \mathrm{OH}$, seja por sua decomposição catalítica na presença de íons metálicos ou de óxidos semicondutores, ou por irradiação com luz ultravioleta (UV). Os radicais • OH são extremamente reativos e fortes agentes oxidantes $\left(E^{\circ}=2,8 \mathrm{~V}\right)$, capazes de mineralizar contaminantes orgânicos por reações sucessivas de oxidação (GOGATE; PANDIT, 2004), podendo, inclusive, em alguns casos, originarem intermediários ou subprodutos mais tóxicos do que os compostos originais. Daneshvar, Behnajady e Asghar (2007) e Nogueira et al(2007) relatam que a geração de radicais hidroxilos na presença de um substrato orgânico pode ocorrer por três vias distintas: (1) abstração de hidrogênio; (2) adição eletrofílica e (3) transferência de elétrons. Entre os diversos mecanismos de oxidação avançada, destacam-se os processos: Fenton $\left(\mathrm{Fe}^{2+} / \mathrm{H}_{2} \mathrm{O}_{2}\right)$, de ozonização $\left(\mathrm{O}_{3}\right)$, com radiação UV, com $\mathrm{H}_{2} \mathrm{O}_{2}$ e a fotocatálise heterogênea usando $\mathrm{TiO}_{2}$ anatase e $\mathrm{ZnO}$ como óxidos ativos.

Particularmente sobre a oxidação de corantes aquosos, a literatura é vasta e, em muitos trabalhos, se relata a elevada eficiência dos processos oxidativos citados anteriormente na degradação de moléculas, como os corantes reativos red 120, yellow 84, black 5 (NEAMTU et al, 2002), índigo carmim (VAUTIER; GUILLARD; HERRMANN, 2001), acido orange 7 (AZAM; HAMID, 2006) e reativo orange 16 (BILGI, DEMIR, 2005). Domínguez, Béltran e Rodríguez (2005) investigaram a combinação de POAs na degradação de efluentes aquosos contendo corante ácido red 88 (ácido 4-(2) hidróxi-1-naftilazo-1-naftenossulfônico). Os autores relacionam a execução de 21 processos de oxidação em escala laboratorial com acompanhamento da remoção de DQO e de carbono orgânico total (COT). Para os três grupos de processos investigados - catálise e fotocatálise homogênea, reações com aplicação de ozônio e fotocatálise heterogênea usando $\mathrm{TiO} 2$ - os resultados obtidos mostraram que os processos foto-Fenton-ozônio (UV/Fe3+/H2O2/O3) e catalítico heterogêneo (O3/TiO2) são mais eficientes na mineralização do corante red 88. Dados cinéticos mostraram ainda que a taxa global de reação se apresenta como uma combinação dos efeitos de degradação por radiação (fotólise) e oxidação por radicais livres.

As principais reações químicas em cada caso estão representadas nas Equações 1 a 7 (BELTRAN; ENCINAR; GONZALÉZ, 1997). Em adição, diversas outras informações tecnológicas importantes sobre os POAs podem ser encontradas nos trabalhos de Hao, Kim e Chiang (2000), Ledakowicz, Solecka e Zylla (2001), Forgacs, Nansheng e Helin (2004) e Wojnárovits e Takács (2008)

Fenton: $\mathrm{Fe}^{2+}+\mathrm{H}_{2} \mathrm{O}_{2} \rightarrow \mathrm{Fe}^{3+}+\cdot{ }^{\bullet} \mathrm{OH}+\mathrm{OH}^{-}$

Equação 1

$\mathrm{R}+\cdot \mathrm{OH} \rightarrow$ produto oxidado

Equação 2

Fotólise: R' + hv $\rightarrow$ R'

Equação 3

$\mathrm{R}^{\prime \bullet} \rightarrow \mathrm{R}^{\prime}$

Equação 4

$\mathrm{R}^{\bullet} \rightarrow$ produto oxidado

Equação 5

Degradação $\mathrm{H}_{2} \mathrm{O}_{2}+\mathrm{h} v \rightarrow 2 \cdot \mathrm{OH}$

Equação 6

$\mathrm{UV} / \mathrm{H}_{2} \mathrm{O}_{2}: \mathrm{R}^{\prime \prime}+\cdot \mathrm{OH} \rightarrow$ produto oxidado

Equação 7

Particularmente, este trabalho visa estudar a descoloração de corantes industriais em solução aquosa sintética ou proveniente de um efluente de lavanderia (efluente têxtil). Os corantes índigo carmim (classe indigóide) e vermelho congo (classe azo) são moléculas modelos que podem facilmente representar efluentes industriais mais complexos. Os processos oxidativos aplicados envolvem as tecnologias Fenton, fotolítica e com UV/ $\mathrm{H}_{2} \mathrm{O}_{2}$, as quais, em geral, são de menores custos, de mais fácil aplicação e com boa eficiência em condições mais brandas de reação. 


\section{Material e métodos}

\section{Reagentes}

Os corantes comerciais índigo carmim (sal dissódico do ácido 5,5 índigo dissulfônico, classe indigóide sulfonato, CI: 73015) e vermelho congo (sal dissódico do ácido benzidinodiazo-bis-1-naftilamina-4-sulfônico, classe diazo sulfonato, CI: 22120) possuem grau analítico; eles foram fornecidos pelo fabricante $\operatorname{Vetec}^{\circledR}$ e usados sem qualquer processo de purificação prévia. As estruturas moleculares dessas espécies estão representadas na Figura 1. Experimentalmente, foram preparadas soluções aquosas (efluentes sintéticos) dos corantes para uma concentração inicial de $20 \mathrm{mg} / \mathrm{L}$ - respectivamente 43 e $29 \mu \mathrm{mol} / \mathrm{L}$ de índigo carmim e vermelho congo. Água ultrapura (condutividade $0,055 \mathrm{mS} / \mathrm{cm}$ ) proveniente de uma unidade UHQPS-MK3 Elga foi usada na dissolução dos corantes. O efluente industrial foi obtido da unidade de tratamento de uma lavanderia industrial localizada na zona metropolitana de Fortaleza, Ceará. O efluente têxtil foi acidificado após sua coleta $(\mathrm{pH}=3)$ e filtrado para fins de conservação. Peróxido de hidrogênio ( 30\%) foi utilizado como espécie química oxidante e fonte de radicais hidroxilos, enquanto que o sulfato ferroso heptahidratado $\left(\mathrm{FeSO}_{4} .7 \mathrm{H}_{2} \mathrm{O}\right)$ foi utilizado como única fonte de íons ferrosos na reação tipo Fenton. No ajuste de $\mathrm{pH}$ das soluções e do efluente foram empregadas soluções 0,5 M de ácido clorídrico ou hidróxido de sódio, conforme o caso.

\section{Experimentos de oxidação Fenton, fotolítica e com UV/ $/ \mathrm{H}_{2} \mathrm{O}_{2}$}

Todos os experimentos de descoloração (degradação) dos efluentes aquosos sintéticos e têxtil foram executados em regime de batelada e realizados em temperatura ambiente $\left(27^{\circ} \mathrm{C}\right)$. No processo oxidativo do tipo Fenton, foram utilizados Erlenmeyers de $125 \mathrm{~mL}$ recobertos com papel laminado para evitar exposição à luz solar. $\mathrm{O}$ volume reacional adotado foi de $100 \mathrm{~mL}$. O pH para as soluções aquosas foi de aproximadamente 6,2 (pH natural) para ambos os corantes, enquanto que, para o efluente, o pH foi ajustado a 3,0. Toda a reação foi executada sob moderada agitação (200 rpm). Na degradação dos corantes, foram utilizadas quantidades de $15 \mathrm{mg}$ de $\mathrm{FeSO}_{4} .7 \mathrm{H}_{2} \mathrm{O}(0,54 \mathrm{mM})$ e $70 \mu \mathrm{L}$ de $\mathrm{H}_{2} \mathrm{O}_{2}$ $30 \%$ (7,05 mM), enquanto que, para o efluente real, foram empregados $60 \mathrm{mg}$ do sal de ferro $(2,16 \mathrm{mM})$ e $750 \mu \mathrm{L}$ do peróxido de hidrogênio concentrado $(75,5 \mathrm{mM})$. As massas de sal e o volume de peróxido de hidrogênio foram definidos em ensaios preliminares de otimização das quantidades dos reagentes Fenton. Nos estudos de descoloração fotooxidativa, foi montado um reator horizontal cilíndrico em PVC (comprimento de $14,7 \mathrm{~cm}$ e diâmetro $2,5 \mathrm{~cm}$ ) com volume reacional útil de $70 \mathrm{~mL}$, possuindo uma lâmpada de vapor de mercúrio (8W, $\lambda$ de $200 \mathrm{a}$ $280 \mathrm{~nm}$ ) acoplada de uma extremidade a outra ao longo do seu corpo, gerando uma intensidade luminosa de aproximadamente $0,064 \mathrm{~W} / \mathrm{cm}^{2}$.

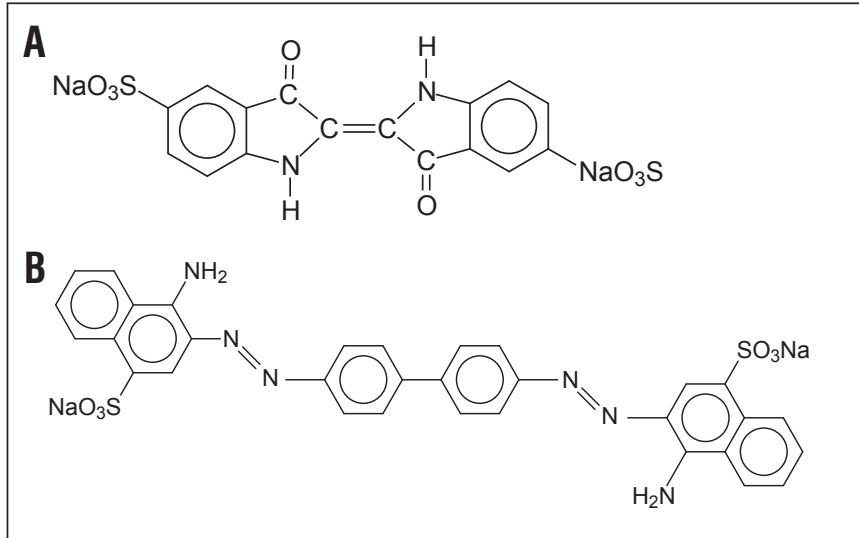

Figura 1 - Estruturas moleculares dos corantes usados nos processos oxidativos (A) índigo carmim e (B) vermelho congo

Dentre os processos fotoassistidos por luz UV, o tratamento combinado com UV/ $\mathrm{H}_{2} \mathrm{O}_{2}$ fez uso de $35 \mu \mathrm{L} \mathrm{H}_{2} \mathrm{O}_{2} 30 \%(5,03 \mathrm{mM})$ para os corantes têxteis sintéticos e $250 \mu \mathrm{L}$ ( $36 \mathrm{mM}$ ) para o efluente industrial, enquanto que, na via fotolítica, utilizou-se somente a fonte de radiação UV para oxidação dos compostos poluentes. Para os diferentes processos reacionais descritos acima, os ensaios de oxidação foram realizados em triplicata, e foram estimados os desvios médios relativos $(\sigma)$ para cada parâmetro analisado. Os valores máximos de desvio relativo $\left(\sigma_{\max }\right)$ adotados são da ordem de 3\%. Em todos os experimentos, foram retiradas alíquotas em tempos pré-determinados de 5, 10, 15, 20, 25 e 30 minutos, as quais foram alcalinizadas com $\mathrm{NaOH} \mathrm{0,5} \mathrm{M} \mathrm{(para} \mathrm{pa-}$ ralisar a reação Fenton) e mantidas em repouso por 30 minutos para precipitação de todo o material em suspensão. A fase sobrenadante foi posteriormente filtrada em membrana de acetato de celulose $(0,45 \mu \mathrm{m})$ e a análise foi realizada.

\section{Análises}

Os estudos cinéticos e de eficiência de descoloração foram realizados a partir das análises espectrofotométricas das alíquotas filtradas, utilizando-se as absorbâncias a $500 \mathrm{~nm}$ (cor real) das amostras centrifugadas (alíquotas) medidas em espectrofotômetro UV-Vis Shimadzu 1601 PC. O comprimento de onda de $500 \mathrm{~nm}$ foi adotado a partir dos espectros moleculares dos corantes sintéticos que apresentam uma banda larga de absorção entre 480 e 600 nm e maior absorbância neste valor. O comprimento de onda de referência é também coincidente com aquele estabelecido pelo Standard methods for the examination of water and wastewater (APHA/AWWA/WEF, 1998) para caracterização de cor em efluentes azul-púrpura (400-530 nm). A concentração do corante remanescente foi calculada segundo a Equação 8:

$\frac{C_{i}}{C_{0}} \times 100=\frac{a_{i}}{a_{0}} \times 100$

Equação 8 
onde:

$\mathrm{C}_{\mathrm{i}}$ denota a concentração de corante em um tempo qualquer de amostragem;

$\mathrm{C}_{0}$ é a concentração inicial de corante;

$\mathrm{a}_{\mathrm{i}}$ e $\mathrm{a}_{0}$ são as absorbâncias a $500 \mathrm{~nm}$ correspondentes a $C_{\mathrm{i}}$ e $C_{0}$, respectivamente.

De forma similar, a eficiência de descoloração foi estimada a partir das absorbâncias medidas segundo a Equação 9:

de descoloração (\%) $=\left(1-\frac{\mathrm{a}_{\mathrm{i}}}{\mathrm{a}_{0}}\right) \times 100$

Equação 9

onde:

$a_{0}$ é a absorbância inicial das soluções coloridas;

$a_{i}$ é a absorbância final das amostras em um tempo reacional qualquer.

A caracterização mais detalhada dos efluentes foi realizada para as análises de pH, cor real a $500 \mathrm{~nm}, \mathrm{DQO}$, turbidez e nitrato, conforme as metodologias estabelecidas pela American Public Health Association (APHA/AWWA/WEF, 1998). Para fins comparativos, a formação de produtos secundários decorrente de cada processo oxidativo foi acompanhada por Cromatografia Líquida de Alta Eficiência (do inglês High Performance Liquid Chromatography, HPLC) e por espectroscopia de massa (GC/MS). As condições analíticas usadas envolveram vazão de $1 \mathrm{~mL} / \mathrm{min}$, fase móvel metanol/água (70:30), coluna ODS Metasil ${ }^{\circledR} 15$ $\mathrm{cm}$ x 4,6 mm e comprimento de onda $(\lambda)$ de $210 \mathrm{~nm}$. Neste comprimento de onda, é esperada a absorção da maioria dos subprodutos formados na degradação dos compostos contendo grupos cromóforos tipo azo e índigo (VAUTIER; GUILLARD; HERRMANN, 2001), por exemplo, os ácidos orgânicos tipo málico, pirúvico, malônico, tartárico, acético, oxálico e os derivados nitrados como o 2-nitrobenzaldeído, o ácido 2-amino benzoico, o nitrobenzeno, o ácido 2-nitrobenzoico, o ácido amino-fumárico, a 2,3-dihidróxi indolina. Intermediários sulfonados são de difícil identificação pela facilidade de conversão (mineralização) do grupo sulfonato a sulfato.

$\mathrm{Na}$ Tabela 1 estão apresentados os resultados da caracterização físico-química para os meios aquosos (sintético e industrial) utilizados nos experimentos de oxidação avançada.

\section{Resultados e discussão}

\section{Cinética da descoloração dos efluentes sintéticos e do efluente têxtil}

Conforme é observado nos diversos tratamentos executados, alguns processos ocorrem de maneira mais rápida do que outros, tornando-se, portanto, interessante investigar a cinética das várias reações estudadas. Segundo Peralta-Hernández et al (2008), a principal evidência de acompanhamento do fenômeno de descoloração refere-se ao desaparecimento da banda de absorção a 487 a $500 \mathrm{~nm}$, que é decorrente da quebra da ligação azo (-N=N-). Em uma primeira aproximação, a taxa global de reação $\left(r_{T}\right)$ consiste na contribuição dos processos de fotólise direta $\left(r_{P}\right)$ e da oxidação por radicais livres $\left(r_{R}\right)$ conforme as Equações 10 e 11 .

$-\mathrm{r}_{\mathrm{T}}=-\left(\mathrm{r}_{\mathrm{P}}+\mathrm{r}\right)_{\mathrm{R}}$

Equação 10

$\mathrm{k}_{\mathrm{T}} \mathrm{C}=\left(\mathrm{k}_{\mathrm{P}}+\mathrm{k}_{\mathrm{R}}\right) \mathrm{C}$ Equação 11

onde:

$r_{T}$ é a taxa global de reação;

$r_{p}$ é a taxa de reação para o processo de fotólise direta;

$r_{R}$ é a taxa de reação para o mecanismo de oxidação por radicais livres.

Com base nesta consideração, os dados cinéticos levantados foram interpretados segundo o modelo linear de primeira ordem (Equação 12) e por um modelo não-linear de pseudoprimeira ordem (Equação 13) relatado anteriormente por Chan e Chu (2003) para descrever a cinética de degradação da atrazina.

$\ln \frac{C_{i}}{C_{O}}=k_{i} t$

Equação 12

onde:

$k_{i}$ é a constante cinética de velocidade de primeira ordem para o processo de oxidação estudado;

$k_{i}=k_{T}$ na interpretação do mecanismo em presença de $\mathrm{UV} / \mathrm{H}_{2} \mathrm{O}_{2}$;

$k_{i}=k_{P}$ quando da oxidação fotolítica.

Tabela 1 - Caracterização das soluções sintéticas e do efluente têxtil usados nos processos de descoloração oxidativa

\begin{tabular}{lccccc} 
Amostra & Cor real (abs) & $\mathrm{pH}$ & Turbidez (NTU) & $\begin{array}{c}\mathrm{DQO} \\
\left(\mathrm{mg} \mathrm{O}_{2} / \mathrm{L}\right)\end{array}$ & $\begin{array}{c}\text { Nitrato } \\
\left(\mathrm{mg} \mathrm{NO}_{3} / \mathrm{L}\right)\end{array}$ \\
Índigo carmim & 0,273 & 6,2 & $N A^{*}$ & 14.463 & 0,0 \\
Vermelho congo & 0,492 & 6,2 & $N A$ & 14.925 & 0,0 \\
Efluente têxtil & 0,181 & 3,0 & 228,8 & 1.437 & 0,315 \\
\hline
\end{tabular}

*NA: não avaliado 
$\frac{\mathrm{C}_{\mathrm{i}}}{\mathrm{C}_{\mathrm{O}}}=1-\frac{\mathrm{t}}{\rho+\sigma \mathrm{t}}$

Equação 13

onde:

$C_{i}$ é a concentração do produto remanescente no sistema após um tempo reacional $t$ ( $\mathrm{min})$;

$C_{0}$ é a concentração inicial do poluente orgânico.

Os parâmetros $\rho$ e $\sigma$ são duas constantes experimentais relacionadas à cinética reacional (min) e à capacidade de oxidação (adimensional) das moléculas oxidadas, respectivamente.

Após procedimentos de derivação da Equação 13, foi obtida a constante $1 / \rho$ como correspondente à constante cinética inicial de pseudoprimeira ordem do sistema reacional e $1 / \sigma$ como a máxima capacidade de oxidação nas diferentes condições investigadas.

Os dados experimentais da cinética de descoloração dos corantes para os três processos oxidativos aplicados segundo os modelos indicados estão apresentados na Figura 2, enquanto os resultados comparativos das respectivas eficiências de remoção de cor e degradação da matéria orgânica em cada caso podem ser visualizados na Tabela 2. Particularmente, o processo Fenton não foi incluído na modelagem clássica de primeira ordem (modelo linear), e sim somente no modelo nãolinear de Chan e Chu (2003) (Equação 13) que descreve uma condição mais homogênea de reação para os momentos iniciais da cinética.

Na Tabela 2, observa-se remoção total de cor (100\%) para ambos os corantes, segundo o processo Fenton $\left(\mathrm{Fe}^{2+} / \mathrm{H}_{2} \mathrm{O}_{2}\right)$, em um tempo reacional de apenas três minutos. Entre todos os tratamentos, o processo Fenton foi o mais eficiente quando combinados os efeitos de descoloração e remoção de matéria orgânica (em termos de DQO). O mecanismo de degradação do tipo fotolítico removeu cerca de $96,7 \%$ de cor para o corante índigo carmim em tempo de 12 minutos (Figura 2), enquanto que, para o corante vermelho congo, a remoção chegou somente a 94,5\% em 40 minutos de reação. No processo fotoquímico, a descoloração foi total no corante índigo carmim em cinco minutos de reação e, para o corante vermelho congo, a remoção de cor atingiu 96,9\% no mesmo tempo. Esses resultados confirmam a elevada reatividade química dos corantes azo e indigóide em todos os processos estudados, embora o tempo reacional seja muito maior para o mecanismo fotolítico - cerca de seis a dez vezes superior ao dos demais processos. A associação de sistemas oxidantes representados pela luz UV e peróxido de hidrogênio ( $\mathrm{UV}+\mathrm{H}_{2} \mathrm{O}_{2}$ ) mostrou-se bastante eficiente, permitindo altas taxas de degradação com consumos muito menores do agente químico $\left(\mathrm{H}_{2} \mathrm{O}_{2}\right)$. Esses resultados mostram boa concordância com aqueles relatados por Neamtu et al (2002), Xu et al (2004) e Behnajady, Modirshahla e Fathi (2006).

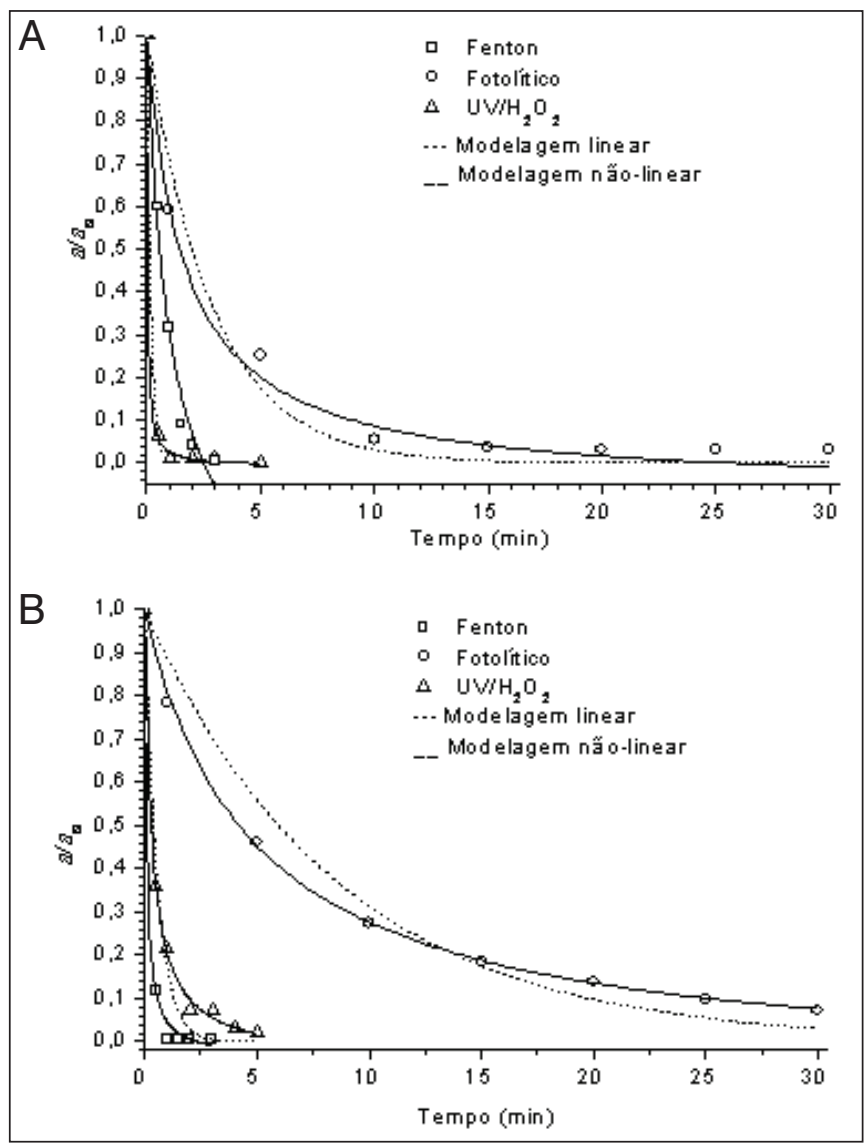

Figura 2 - Cinética reacional para degradação dos corantes índigo carmim (A) e vermelho congo (B) pelos processos Fenton, fotolítico e com $\mathrm{UV} / \mathrm{H}_{2} \mathrm{O}_{2} \cdot \mathrm{C}_{0}=20 \mathrm{ppm}$ e $\mathrm{T}=27^{\circ} \mathrm{C}$

Tabela 2 - Descoloração (\%) e constantes cinéticas segundo modelo linear para a oxidação avançada dos efluentes aquosos sintéticos (índigo carmim e vermelho do congo) e têxtil $\left(T=27^{\circ} \mathrm{C}\right)$

\begin{tabular}{|c|c|c|c|c|c|c|}
\hline Amostra & $\begin{array}{l}\text { Processo } \\
\text { oxidativo }\end{array}$ & $\begin{array}{c}\text { Remoção de } \text { cor }^{\alpha} \\
(\%)\end{array}$ & $\begin{array}{c}\text { Remoção de } \\
\operatorname{DQO}^{\beta}(\%)\end{array}$ & $\mathrm{k}_{\mathrm{T}} \times 10\left(\mathrm{~min}^{-1}\right)$ & $\mathrm{k}_{\mathrm{p}} \times 10\left(\mathrm{~min}^{-1}\right)$ & $k_{R} \times 10^{d}\left(\min ^{-1}\right)$ \\
\hline \multirow{3}{*}{ Índigo carmim } & Fenton $^{\mathrm{a}}$ & 100 & 95 & - & - & - \\
\hline & $\mathrm{UV} / \mathrm{H}_{2} \mathrm{O}_{2}^{\mathrm{b}}$ & 100 & 89 & 54,4 & - & \multirow{2}{*}{50,9} \\
\hline & Fotolítico & 98 & 80 & - & 3,5 & \\
\hline \multirow{3}{*}{ Vermelho congo } & Fenton & 100 & 94 & - & - & - \\
\hline & $\mathrm{UV} / \mathrm{H}_{2} \mathrm{O}_{2}$ & 97 & 85 & 17,6 & - & \multirow{2}{*}{16,4} \\
\hline & Fotolítico & 90 & 70 & - & 1,2 & \\
\hline \multirow{3}{*}{ Efluente têxtil } & Fenton & 99 & 50 & - & - & - \\
\hline & $\mathrm{UV} / \mathrm{H}_{2} \mathrm{O}_{2}$ & 99 & 33 & 1,02 & - & \multirow{2}{*}{0,87} \\
\hline & Fotolítico & 64 & 17 & - & 0,15 & \\
\hline
\end{tabular}

a após 3 minutos; ' ${ }^{\mathrm{a}}$ após 5 minutos; ${ }^{\mathrm{c}}$ após 30 minutos; ${ }^{\mathrm{d}} \mathrm{k}_{\mathrm{R}}=\mathrm{k}_{\mathrm{T}}-\mathrm{k}_{\mathrm{P}} ;{ }^{a} \sigma_{\text {máx }}=1,23 \% ;{ }^{\beta} \sigma_{\text {máx }}=2,39 \%$; - não avaliado. 
Tabela 3 - Parâmetros cinéticos segundo modelo não-linear para a descoloração das soluções aquosas contendo os corantes sintéticos e para 0 efluente têxtil

\begin{tabular}{|c|c|c|c|c|c|c|}
\hline & \multicolumn{2}{|c|}{ Fenton } & \multicolumn{2}{|c|}{ Fotolítico } & \multicolumn{2}{|c|}{$\mathrm{UV} / \mathrm{H}_{2} \mathrm{O}_{2}$} \\
\hline & $1 / \rho\left(\min ^{-1}\right)$ & $1 / \sigma$ & $1 / \rho\left(\min ^{-1}\right)$ & $1 / \sigma$ & $1 / \rho\left(\min ^{-1}\right)$ & $1 / \sigma$ \\
\hline Índigo carmim & 1,36 & 1,42 & 0,65 & 1,06 & 28,5 & 1,01 \\
\hline Vermelho congo & 12,1 & 1,04 & 0,22 & 1,07 & 3,30 & 1,04 \\
\hline Efluente têxtil & 9,02 & 0,99 & 0,18 & 1,04 & 0,01 & 5,12 \\
\hline
\end{tabular}

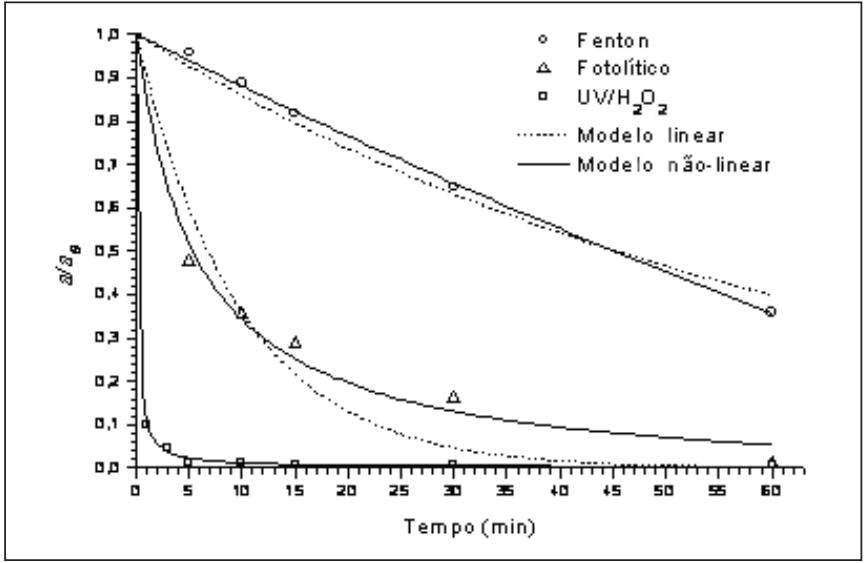

Figura 3 - Cinética reacional para descoloração do efluente têxtil $\left(\mathrm{T}=27^{\circ} \mathrm{C}\right.$ ) segundo os processos Fenton, fotolítico e com UV/ $\mathrm{H}_{2} \mathrm{O}_{2}$

Adicionalmente, as modelagens cinéticas propostas tanto pelo modelo linear de primeira ordem quanto pelo modelo não-linear de pseudoprimeira ordem (Equações 12 e 13) permitiram calcular os principais parâmetros cinéticos para cada processo oxidativo investigado, conforme a Tabela 3, os quais apontam a elevada eficiência dos processos contendo $\mathrm{H}_{2} \mathrm{O}_{2}$ na degradação de azo corantes. Os valores de remoção de DQO são elevados, embora a mineralização não tenha sido completa, pois produtos secundários foram eventualmente formados. O processo fotolítico mostrou-se menos eficiente entre os processos estudados. Para o efluente têxtil, a eficiência de descoloração se opõe às reduzidas taxas de degradação da matéria orgânica (Figura 3), principalmente em função da diversidade e da complexidade dos compostos inicialmente presentes.

Do ponto de vista cinético, as constantes cinéticas de pseudoprimeira ordem $\left(k_{T}, k_{\mathrm{p}}, k_{\mathrm{R}}\right)$ mostraram a maior reatividade do corante índigo frente ao vermelho congo com valores cerca de três vezes superiores aos obtidos no processo fotolíticos e com $\mathrm{UV} / \mathrm{H}_{2} \mathrm{O}_{2}$. As diferenças são ainda maiores quando comparadas ao efluente têxtil (mistura complexa de compostos orgânicos), no qual os valores de $k_{T}$ (fotólise + radicais livres) e $k_{R}$ (radicais livres) são até 50 vezes menores, considerando-se a mesma eficiência de descoloração. Essas diferenças refletem a importância da concentração de radicais ${ }^{\bullet} \mathrm{OH}$ disponíveis para o sistema reacional $\left(k_{R} \cong k_{T} \gg>k_{\mathrm{P}}\right)$, conforme já observado por Peralta-Hernández et al (2008).
Tabela 4 - Caracterização físico-química do efluente têxtil após tratamento segundo os processos Fenton, fotolítico e com UV/ $\mathrm{H}_{2} \mathrm{O}_{2}$

\begin{tabular}{lccc} 
& \multicolumn{3}{c}{ Tratamento } \\
\cline { 2 - 4 } Variável & Fenton & Fotolítico & $\mathbf{U V} / \mathbf{H}_{2} \mathbf{O}_{2}$ \\
\hline Cor aparente $(\mathrm{abs})$ & 0,004 & 0,065 & 0,007 \\
$\mathrm{pH}$ & 2,39 & 3,69 & 3,71 \\
\hline Turbidez $(\mathrm{NTU})$ & 14,3 & 13,7 & 3,65 \\
\hline $\mathrm{DQO}\left(\mathrm{mg} \mathrm{O}_{2} / \mathrm{L}\right)$ & 458 & 763 & 610 \\
\hline Nitrato $\left(\mathrm{mg} \mathrm{NO}_{3}^{-} / \mathrm{L}\right)$ & 2,042 & 0,336 & 2,391 \\
\hline
\end{tabular}

Os resultados simulados da aplicação do modelo não-linear de Chan e Chu (2003) aos dados experimentais estão apresentados na Tabela 3.

Em geral, os resultados obtidos corroboram com os estimados na modelagem anterior (linear). Particularmente para o corante índigo carmim, a cinética reacional foi mais favorável para o processo com $\mathrm{UV} / \mathrm{H}_{2} \mathrm{O}_{2}$. O valor de constante cinética $(1 / \rho)$ no processo de descoloração do corante índigo carmim com UV/ $\mathrm{H}_{2} \mathrm{O}_{2}$ é cerca de 20 a 40 vezes superior àqueles observados nos demais processos. Para o corante vermelho congo (azo), o processo Fenton foi muito mais eficiente. No que diz respeito à capacidade de oxidação $(1 / \sigma)$, as reatividades observadas são muito semelhantes para ambos os corantes sintéticos, com valores variando entre 1,01 e 1,42.

\section{Caracterização final do efluente e avaliação dos produtos formados}

A Tabela 4 apresenta a caracterização final do efluente têxtil após os tratamentos oxidativos empregados.

Adicionalmente, para os três efluentes investigados, foi realizado um estudo adicional da eficiência de degradação pelo acompanhamento da formação de nitrato. As curvas cinéticas levantadas para os diferentes mecanismos oxidativos estão apresentadas nas Figuras 4 e 5 .

Conforme observado, os processos Fenton e com UV/ $\mathrm{H}_{2} \mathrm{O}_{2}$ foram aqueles que determinaram a maior concentração de nitrato ao final da reação (maior descoloração e mineralização) para ambos 


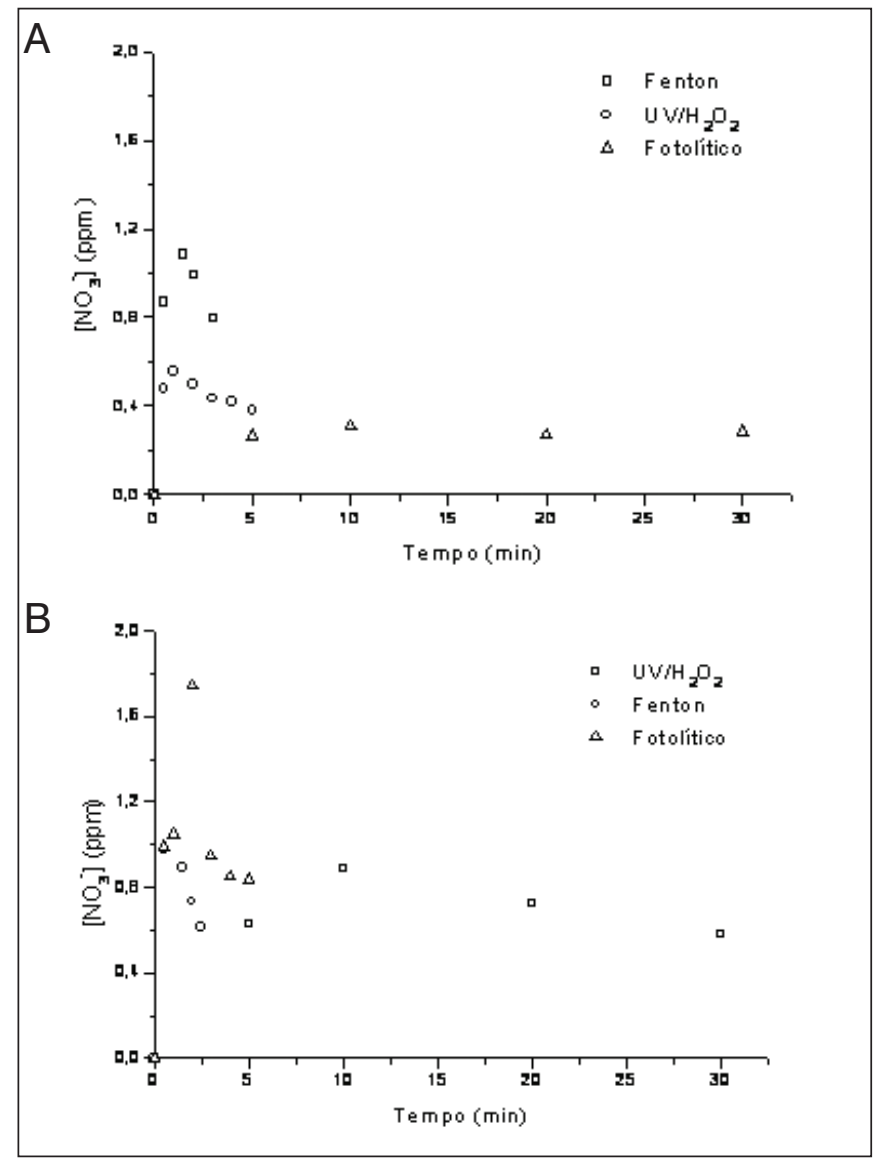

Figura 4 - Cinética reacional de formação de nitrato nas soluções aquosas de corantes sintéticos $(A)$ índigo carmim e (B) vermelho congo para os diferentes processos oxidativos estudados $\left(T=27^{\circ} \mathrm{C}\right)$

os efluentes (aquoso sintético e efluente têxtil). Em especial, a concentração do íon nitrato está diretamente relacionada aos processos redox sobre as ligações $-\mathrm{N}=\mathrm{N}$ - e, considerando as concentrações iniciais, pode-se esperar que não tenha ocorrido a conversão total dos intermediários nitrados produzidos ao longo dos diferentes mecanismos oxidativos empregados e, ainda, uma eventual decomposição do nitrato nas condições reacionais dos meios em estudo. A análise por HPLC/GC-MS das misturas finais de reação revela composições bastante semelhantes e com indicativo de formação de um pequeno número de subprodutos. Em geral, para as soluções aquosas de índigo carmim e vermelho congo, segundo os processos Fenton e com $\mathrm{UV} / \mathrm{H}_{2} \mathrm{O}_{2}$, foram identificados compostos em tempos de retenção $\left(t_{R}\right)$ mais curtos: $\mathrm{t}_{\mathrm{R} 1}=1,56 ; \mathrm{t}_{\mathrm{R} 2}=1,63$ e $\mathrm{t}_{\mathrm{R} 3}=1,68$, relacionados ao ácido acético, ao ácido 3-nitropropiônico e ao ácido oxálico, respectivamente (VAUTIER; GUILLARD; HERRMANN, 2001). Para a rota fotolítica, nenhum coproduto foi identificado nas condições da análise

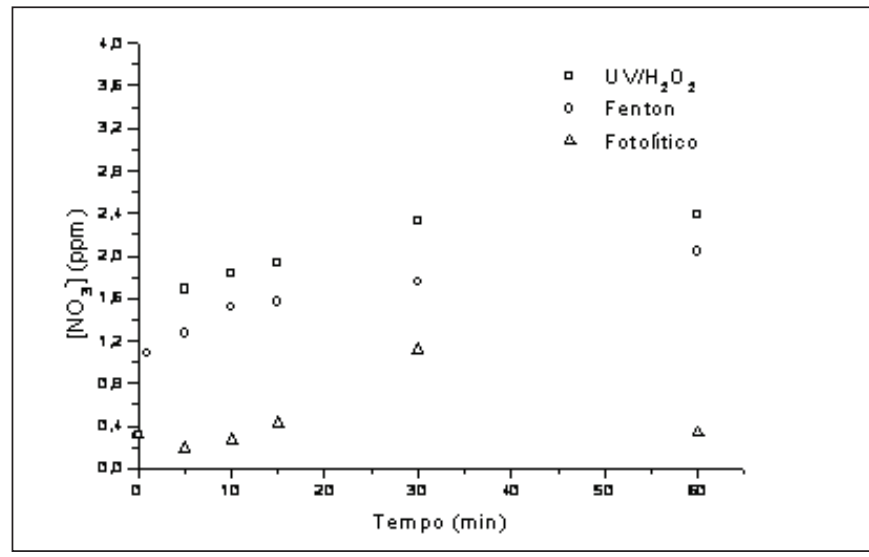

Figura 5 - Cinética reacional de formação de nitrato no efluente têxtil para os diferentes processos oxidativos avançados em estudo $\left(T=27^{\circ} \mathrm{C}\right)$

realizada. No caso do efluente têxtil, os resultados cromatográficos são também muitos semelhantes e, para todos os processos aplicados, os subprodutos principais identificados referem-se aos ácidos acético $\left(t_{R 1}=1,29\right)$, 3-nitropropiônico $\left(t_{R 2}=1,49\right)$, oxálico $\left(t_{R 3}=1,58\right)$, 4-nitro-3-oxobutírico $\left(\mathrm{t}_{\mathrm{R} 4}=1,79\right)$ e ftálico, $\left(\mathrm{t}_{\mathrm{R} 5}=1,90\right)$.

\section{Conclusões}

Os processos de oxidação avançada mostraram-se bastantes eficientes na descoloração/degradação das soluções corantes e do efluente têxtil industrial, permitindo eliminar por completo (branqueamento) a cor dos meios aquosos sintéticos pelo processo Fenton em apenas três minutos e em até cinco minutos por meio de oxidação com luz UV e peróxido $\left(\mathrm{UV} / \mathrm{H}_{2} \mathrm{O}_{2}\right)$. No efluente, o melhor resultado de descoloração ( 99\%) foi obtido segundo a reação Fenton em apenas três minutos, enquanto o processo fotoquímico removeu o mesmo percentual de cor em cinco minutos de reação. Com relação ao grau de mineralização do efluente, pode-se constatar uma maior redução do teor de matéria orgânica, via remoção de $\mathrm{DQO}$, por meio dos processos Fenton e UV/ $\mathrm{H}_{2} \mathrm{O}_{2}$ (aproximadamente 50 e $33 \%$, respectivamente), enquanto que, no mecanismo fotolítico, o grau de mineralização do efluente foi de apenas 17\%. Estes resultados confirmam a maior resistência à oxidação deste material, principalmente em função de sua complexidade composicional. No que diz respeito à formação de nitrato a partir das soluções aquosas sintéticas de corantes, os processos aplicados determinam o aumento de concentração deste subproduto inorgânico, embora os valores obtidos sejam relativamente inferiores aos esperados estequiometricamente. 


\section{Referências}

APHA/AWWA/WEF. Standard methods for the examination of water and wastewater. Washington, DC:APHA, 1998.

AZAM, A.; HAMID, A. Effects of gap size and UV dosage on decolorization of $C$. I. acid orange 7 by $U \mathrm{~V} \mathrm{H}_{2} \mathrm{O}_{2}$ process. Journal of Hazardous Materials, v. 133, p. 167-171, 2006.

BEHNAJADY, M.A.; MODIRSHAHLA, N.; FATHI, H. Kinetics of decolorization of an azo dye in UV alone and $\mathrm{UV} / \mathrm{H}_{2} \mathrm{O}_{2}$ processes. Journal of Hazardous Materials, v. 136, p. 816-821, 2006.

BELTRAN, F.J.; ENCINAR, J.M; GONZALÉZ, J.F. Industrial wastewater advanced oxidation. Part 2. Ozone combined with hydrogen peroxide or UV radiation. Water Research, v. 31, n. 10, p. 2415-2428, 1997

BILGI, S.; DEMIR, C. Identification of photooxidation degradation products of C. I. Reactive Orange 16 dye by gas chromatography-mass spectrometry. Dyes and Pigments, v. 66, p. 69-76, 2005.

CHAN, K.H.; CHU, W. Modeling the reaction kinetics of Fenton's process on the removal of atrazine. Chemosphere, v. 51, n. 4, p. 305311,2003

COUGHLIN, M.F.; KINKLE, B.K.; BISHOP, P.L. High performance degradation of azo dye Acid Orange 7 and sulfanilic acid in a laboratory scale reactor after seeding with cultured bacterial strains. Water Research, v. 37, n. 11, p. 2757-2763, 2003.

DANESHVAR, N.; BEHNAJADY, M.A.; ASGHAR, Y.Z. Photooxidative degradation of 4-nitrophenol (4-NP) in $\mathrm{UV} / \mathrm{H}_{2} \mathrm{O}_{2}$ process: Influence of operational parameters and reaction mechanism. Journal of Hazardous Materials, v. 139, p. 275-279, 2007

DOMÍNGUEZ, J.R.; BÉLTRAN, J.; RODRÍGUEZ, O. Vis and UV photocatalytic detoxification methods (using $\mathrm{TiO}_{2}, \mathrm{TiO}_{2} / \mathrm{H}_{2} \mathrm{O}_{2}, \mathrm{TiO}_{2} / \mathrm{O}_{3}$, $\mathrm{TiO}_{2} / \mathrm{S}_{2} \mathrm{O}_{8}{ }^{2-}, \mathrm{O}_{3}, \mathrm{H}_{2} \mathrm{O}_{2}, \mathrm{~S}_{2} \mathrm{O}_{8}{ }^{2-}, \mathrm{Fe}^{3+} / \mathrm{H}_{2} \mathrm{O}_{2}$ and $\left.\mathrm{Fe}^{3+} / \mathrm{H}_{2} \mathrm{O}_{2} / \mathrm{C}_{2} \mathrm{O}_{4}{ }^{2-}\right)$ for $d y e s$ treatment. Catalysis today, v. 101, p. 389-395, 2005

FORGACS, E. NANSHENG, D.; HELIN, H. Removal of synthetic dyes from wastewaters: a review. Environmental International, v. 30, n. 7, p. 953-971, 2004

GOGATE, R.; PANDIT, B. A review of imperative technologies for wastewater treatment I: oxidation technologies at ambient conditions. Advances in Environmental Research, v. 8, p. 501-551, 2004.

HAO, O.J.; KIM, H.; CHIANG, P.C. Decolorization of wastewater. Critical Reviews in Environmental Science and Technology, v. 30, n. 4, p. 449$505,2000$.
KUŠIĆ, H. et al. Fenton type processes for minimization of organic content in coloured wastewaters. Part II: Combination with zeolites. Dyes and Pigments, v. 74, p. 388-395, 2007.

LEDAKOWICZ, S.; SOLECKA, M.; ZYLLA, R. Biodegradation, decolourisation and detoxification of textile wastewater enhanced by advanced oxidation processes. Journal of Biotechnology, v. 89, n. 2, p. 175-184, 2001.

NEAMTU, M. et al. Kinetics of decolorization and mineralization of reactive azo dyes in aqueous solution by the $\mathrm{UV} / \mathrm{H}_{2} \mathrm{O}_{2}$ oxidation. Dyes and Pigments, v. 53, p. 93-99, 2002.

NOGUEIRA, R.F.P. et al. Fundamentos e aplicações ambientais dos processos Fenton e foto-Fenton. Química Nova, v. 30, n. 2, p. 400-408 2007

PETERNEL, l.; KOPRIVANAC, N.; KUŠIĆ, H. UV-based processes for reactive azo dye mineralization. Water Research, v. 40, n. 3, p. 525-532, 2006

POULOPOULOS, S.G.; ARVANITAKIS, F.; PHILIPPOPOULOS, C.J. Photochemical treatment of phenol aqueous solutions using ultraviolet radiation and hydrogen peroxide. Journal of Hazardous Materials, v. 129, p. 64-68, 2006.

PERALTA-HERNÁNDEZ, J.M. et al. Comparison of hydrogen peroxidebased processes for treating dye-containing wastewater: Decolorization and destruction of Orange $\|$ azo dye in dilute solution. Dyes and Pigments, v. 76, p. 656-662, 2008

ROTT, U.; MINKE, R. Overview of wastewater treatment and recycling in the textile processing industry. Water Science and Technology, v. 40, n. 1, p. 137-144, 1999

SHU, H.; CHANG, M. Decolorization effects of six azo dyes by $\mathrm{O}_{3}, \mathrm{UV} / \mathrm{O}_{3}$ and $\mathrm{UV} / \mathrm{H}_{2} \mathrm{O}_{2}$ processes. Dyes and Pigments, v. 65, p. 25-31, 2005.

VAUTIER, M.; GUILLARD, C.; HERRMANN, J-M. Photocatalytic degradation of dyes in water: case study of indigo and indigo carmine. Journal of Catalysis, v. 201, n. 1, p. 46-59, 2001.

WOJNÁROVITS, L.; TAKÁCS, E. Irradiation treatment of azo dye containing wastewater: an overview. Radiation Physics and Chemistry, v. 77, p. $225-244,2008$

$\mathrm{XU}, \mathrm{X}$. et al. Degradation of dyes in aqueous solutions by the Fenton process. Chemosphere, v. 57, p. 595-600, 2004.

ZAZO, J.A. et al. Catalytic wet peroxide oxidation of phenol with a Fe/active carbon catalyst. Applied Catalysis B: Environmental, v. 65, p. 261-268, 2006. 